\title{
Dynamic Bandwidth Allocation in LAN using Dynamic Excess Rate Sensing
}

\author{
Muhammad Abubakar Muhammad ${ }^{1}$, Muhammad Azhar Mushatq ${ }^{2}$, Abid Sultan $^{3}$, Muhammad Afrasayab $^{4}$ \\ Department of Computer Sciecne \& IT, University of Sargodha Sub- Campus Bhakkar, Bhakkar, Pakistan ${ }^{1,2,3}$ \\ Department of Computer Sciecne, Govt. College University, Faisalabad, Faisalabad, Pakistan ${ }^{4}$
}

\begin{abstract}
Today human and information processing system both need rapid access to anything they want on the internet. To fulfill these needs more and more internet service providers with a large amount of bandwidth are introducing themselves in the market. For these providers, a lot of bandwidth is free during off-peak hours while during peak hours the total available bandwidth might be insufficient. The primary purpose of our research is to divide and distribute the excessive bandwidth among the users during off-peak hours to attain the maximum user satisfaction. In order to do this dynamic excess rate (DER) scheme and its frame work is proposed in this paper.
\end{abstract}

Keywords-DER; ISPs; PIR; DBA; MRT; CIR

\section{INTRODUCTION}

After wheel and electricity, the Internet has transformed the world into an information village. The provision of the internet is carried out by specialized operators called "Internet Service Provider" (ISP). These ISPs operate on a different level ranging from continental to metropolitan local or ISPs. Internet service providers, regardless of the level on which they are operating, provide both network infrastructure and the bandwidth to both individuals and the corporate world.

The users want the maximum bandwidth at lowest billing, while on other hand the provider has bulk of bandwidth which can be free at off-peak hours. To distribute the bandwidth among a large number of users ISP uses different mechanism most commonly as static allocation at subscribed peak information rate (PIR). In static allocation, the excessive bulks of the bandwidth cannot produce advantages for both the users and providers. Where on the other hand bandwidth eager users want to browse and download at maximum data rates within their budgets. The above scenario arises as a challenge for the local and regional ISPs, trying to full fill the needs of their users with the best data rates.

In order to create a better user experience, dynamic allocation of the excessive available bandwidth through sensing the real-time utilization is the need of time. In this paper, we have proposed a methodology called DER, which will serve the purpose of dynamic allocation of bandwidth through sensing real-time available bandwidth. Moreover, it will also improve the quality of services (QoS), user experience and the business opportunities for providers of a network [1].

In dynamic bandwidth allocation scheme, allocation can be changed dynamically depending on different factors. So far Dynamic Bandwidth Allocation (DBA) is completely adaptable only on OFC (Optical Fiber Cable) networks. In DBA the link capacity is enhanced with extra bandwidth (if available) on per-flow per request basis which can be implemented only by the access router itself [2]. In other word allocation schemes, link capacity is enhanced for a very short period of time, on request, and on a temporary basis [3]. The major benefits of the DBA may include [4].

- Efficient performance in case of over subscription.

- Low latency on both downstream and upstream.

- Strong QoS.

- Increased user satisfaction.

- Fairness in distribution among different users belonging to the same priority/group/class.

However, the two major drawbacks of most of the DBA algorithms are as:

- The allocation of excessive rates is only for a very short period of time even may be for a single flow request.

- Lack of supportability for shared networks like asymmetric digital subscriber line (ADSL), ADSL+, wireless local area network (WLAN) and Internet cables.

In this research, we have tried to address and resolve the above drawbacks of the DBA approach by designing a scheme named as DER that will be able to allocate the excessive bandwidth for a longer period of time and will work fine for all networks.

This paper is ordered as follows. Section II covers the related work whereas Section III presents the proposed methodology. In Section IV, flow charts of the proposed method lay are presented and finally in Section V conclusion and future work is presented.

\section{RELATED WORK}

In literature, different schemes have been proposed by authors for bandwidth management of local area network (LAN) or WLAN. The main objective is to make efficient use of bandwidth while achieving good QoS. In this section, we have covered some of the existing bandwidth management schemes.

An AIFS or contention window based scheme for LAN is proposed by authors in [5]. The scheme is mainly based on 
priority level. This priority level is implemented by using a priority table at the access point, and priority will be assigned to each user on the basis of physical address of the node. Moreover, bandwidth utilization will also be monitored by this technique. In case a user is making improper usage of bandwidth, the bandwidth from that user will be allocated to other high priority users [5].

For users who spend most of their time for downloading videos, a Weighted Fair Intelligent Bandwidth Algorithm (FIBA) has been proposed by Xiaomei Yu et al. FIBA algorithm is computationally less costly, prevent unwanted time delays and make usage of reporting instrument for efficiently running videos traffic. Once the connection is triggered by a switch, FIBA monitors the total available bandwidth and fairly re-allocate the bandwidth. The major fault of FIBA is that it has unlimited downloading and require a lot of configuration [6].

As described in cluster-based Bandwidth Allocation Algorithm, bandwidth can be allocated to clusters and specific users. In a cluster, bandwidth is allotted to the cluster head only, this happens when cluster head obtains content from the source station. The main objective is to obtain the highest throughput in a network connection [7].

In Smart Clustering Based technique, a clever clustering approach is designed for wireless network nodes through which bandwidth will be allocated dynamically to the users. Moreover, this method is useful for wireless nodes to adapt bandwidth allocation according to the changing number of users over time [8].

In order to increase benefits for the user, the authors in [9] designed the Dynamic Bandwidth Allocation algorithm to supervise and control traffic of the network. This algorithm ensures QoS by reducing network congestion and divide bandwidth equally to active network users as well as those users who are not currently active in the network. The main benefit of the algorithm is all users are treated equally within the network. In order to deal with hypermedia data that needs a large range of bandwidth, it uses a multiplexing technique. The major problem of the DBA algorithm is that it has absences of appropriate feedback and reporting instruments. In a situation where a large amount of data is downloaded bandwidth costs will be increased and this method only supplies bandwidth according to the demand [9].

The authors in [10] proposed mesh topology network architecture, based on reformative digital video broadcasting geostationary satellite (DVB-S GEO), which has the ability to connect heterogeneous terrestrial domain name server (DNS) with each other, within lowest delay. In proposed satellite network architecture an appropriate dynamic bandwidth management instrument is applied, that is used to enable the delivery of interactive internet protocol (IP) dependent hypermedia services according to the defined level of QoS [10].

\section{PRoposed Methodology}

This section covers the proposed DER framework for dynamic bandwidth allocation, see Fig. 5. The components of the framework are:

\section{A. Scheduler}

The scheduler in the described flowcharts is a predefined process. The scheduler will perform the following function:

a) Activation of DER: As the aim of designing the DER flowcharts is to distribute the excessive bandwidth when the resources are free, or to withdraw when the network is over populated. The system support personals will decide the peak hours, off-peak hours, and the scheduler will be set accordingly. So the scheduler will automatically activate and deactivate the appropriate action.

b) Running the DER: The second function of the scheduler is to run the pre-activated DER after regular intervals of time. The duration of the intervals will be set by the network administrator, keeping in view the number of users and the hardware capabilities as the running of DER will consume the processing and other resources. The intervals should not be too short to choke the whole system.

\section{B. Active user Count}

The DER mechanism starts with the count of active online subscribers or users. The active user means that the user is using the resources of the network at that time. The total users can be counted by their active IP addresses and also by their usernames.

\section{Count Total Bandwidth usage}

After counting the total number of active users the total usage or the maximum usage of the bandwidth during certain time will be calculated. This will be done by analyzing the wide area network (WAN) interfaces in which backhaul link is terminated. The peak reading of the Multi Router Traffic (MRT) graph can also be taken as an alternative. However, DER can also be applied on the basis of only the active or alive user count. The current usage will be decided from the maximum data rate variable on last 15 minutes (or any other period of time) using Multi Router Traffic Grapher (MRTG) of WAN interfaces. If more than one WAN interface is present the system will simply sum up the average rates.

\section{Check the Actual Available Bandwidth}

The actual bandwidth is not that has been written on your Service Level Agreement (SLA). It is that you actually get when the backhaul link is being fully utilized. If the provider has Committed Information Rate (CIR) backbone links the condition might be similar as written in the agreements but in case of shared links the condition will be different than the actual bandwidth will be remarkably low as written in SLA. In both scenarios, the system will take the maximum MRT value and will consider it the actual network bandwidth. The second option is more suitable in which service provider will manually define the total consumable bandwidth that he has. In both cases, the system will read the variable in which this is stored.

\section{E. Estimate Net Excessive Bandwidth}

In order to find the excessive bandwidth (EB), the difference between the actual available bandwidth (TB) and the total bandwidth usage during the specific time will be used, see equation (1).

$\mathrm{EB}=\mathrm{TB}-$ Max.Usage (in defined period of time) 


\section{F. Calculate Excessive Bandwidth Per user}

In this step, the system will calculate the per-user available excessive data rate (NEB). This will be done by a simple equation as shown in (2).

NEB per user $=\mathrm{EB} /$ Total active user count (AUC)

\section{G. Analyze and Match}

After taking both counts the algorithm will check the predefined thresholds for the implementation of the DER. These thresholds will be set by the service providers depending upon the following factors.

- The total backhaul bandwidth of network

- Total number of subscriber

- Total subscription for bandwidth

- Sharing Ratio

- Number of corporate users

- Number of home users

- Average, minimum and maximum data rates on WAN interfaces

- Peak hours and off-peak hours

After reading the threshold variable the system will analyze and match the thresholds to the E.B per user value. Moreover, the system will decide whether to distribute the excess network bandwidth or not.

$\mathrm{Xc}=\mathrm{E} . \mathrm{B}$ per user - Threshold

By this analysis system will also able to cut down the already allocated data rates in the peak time or in the conditions of over subscriptions.

\section{H. Decide the Action}

After the analysis system will make the allocation decision on the of previously calculated values that decision might be

- Do nothing and wait for next turn

- Deactivate the DER

- Allocate the Excessive rate as per policy

- Withdraw some data rate from active users

\section{Decide the Per-user Allocation}

As it is a strong probability that the more users will be online after taking the values from the user count so it is very important to keep them served and not starved. Thus we cannot allocate all excessive bandwidth. The system will allocate the excess data rate to every online user less than the value of the E.B per user as the something must be available for the new users till the next turn of the DER.

\section{J. Updating the user Queues}

As most of the Radius-based authenticated networks maintain the user queues in which the data rate of the user is also defined. The DER will update the all or the selective queues with an incremental or detrimental data rate.

\section{K. Updating the user Profile}

After the decision of the allocation, the user profile of the active user will be updated and the data rates in the databases will also be changed. In this respect in the database, a separate field of Excess rate might be included which will only be updated by the DER scheme. But here the best approach itself is to just update the queues, not the profiles as the queues are made only for the one connection or even for the session.

\section{Flow Charts of Proposed Methodology}

The proposed methodology of DER consists of four parts. As in Fig. 1 the first part is designed to count the online active users and to estimate the bandwidth usage and availability. It counts the total active subscribers, gets the overall maximum usage of the bandwidth and then performs some calculation to determine the total available excessive rate. This part of the methodology will be used in all other three parts as their action will be decided from the result achieved in part one. There are three possible actions listed as:

a) No allocation.

b) Allocate the excessive bandwidth.

c) Withdraw bandwidth

The second flow chart in Fig. 2 deals with the possibility of no allocation of bandwidth, which occurs when there is not a sufficient bandwidth for the DER to be provided to the active users. This will happen when the number of active user or the usage of the total available bandwidth on the network is very high.

The third flow chart deals with the possibility to allocate the excessive bandwidth. This is the ideal condition or the main goal of our research. On the basis of the result achieved from the first part, the decision will be made of how much additional bandwidth will be allocated to the users. The additional bandwidth will be added up to the already allocated peak information rate (PIR) values in the user queues. This possibility will occur if the number of online users is less than the pre-described threshold and the usage of the bandwidth is low seeing Fig. 3.

The flow chart in Fig. 4 is designed to cope with the worst possibility of withdrawing bandwidth on a network. If the network is over subscribed, then to accommodate the maximum number of active users a small portion of the bandwidth (PIR allocated statically according to user profile) will be cut down. This will avoid the starvation of resources on the network. 


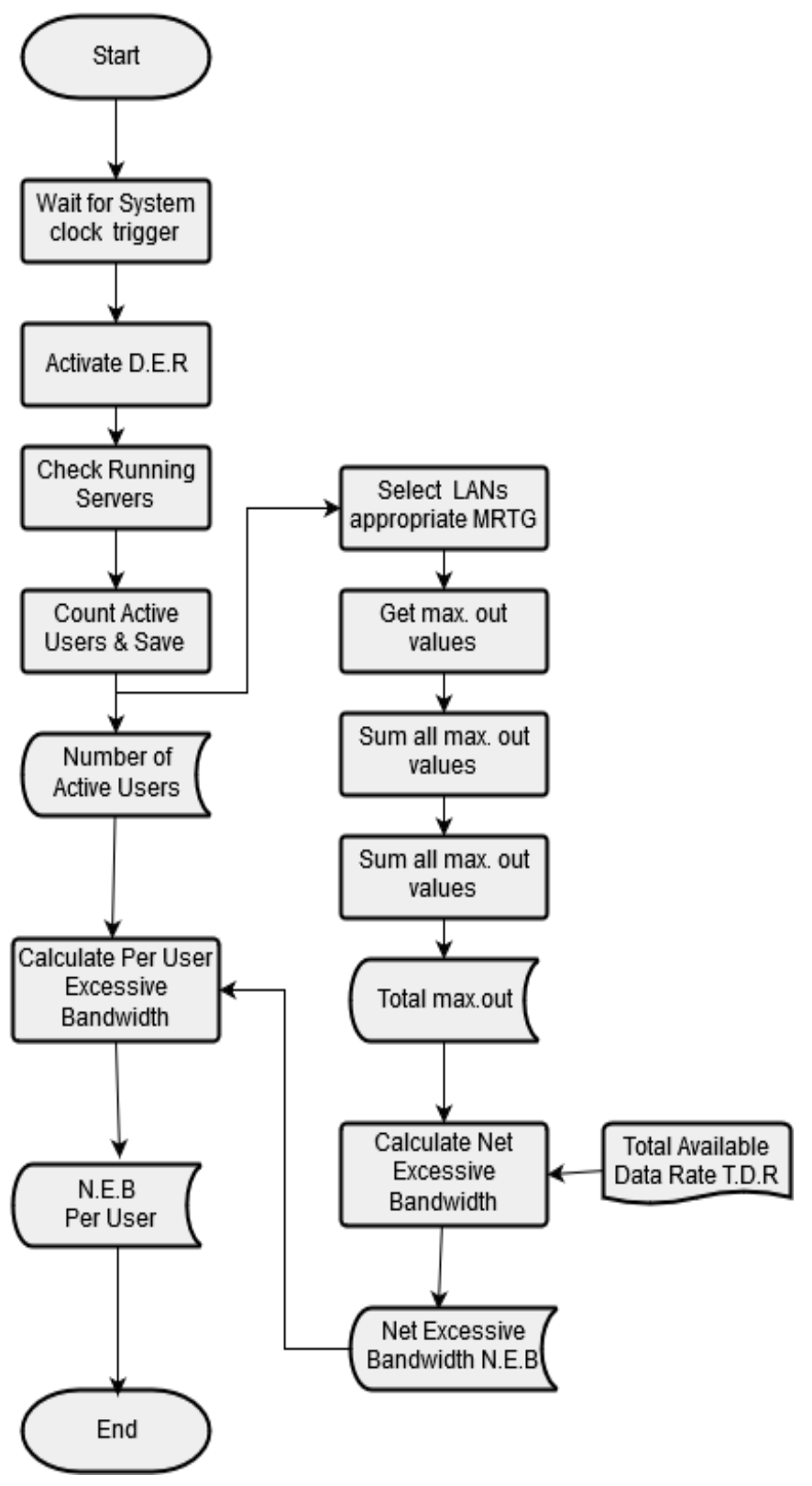

Fig. 1. Flow Chart for Active user Count \& Bandwidth Estimation.

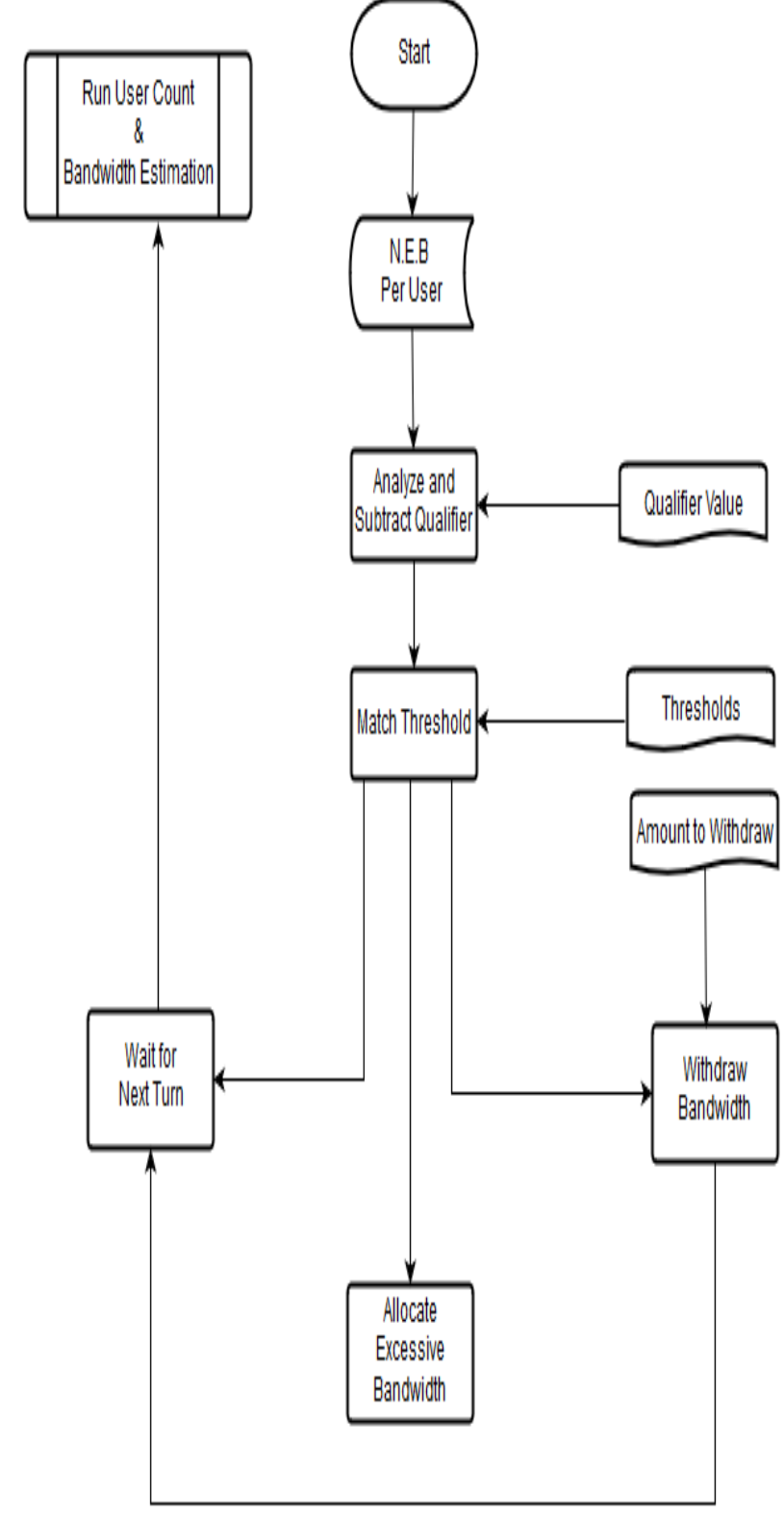

Fig. 2. Flow Chart for Analyzer and Decision Maker. 


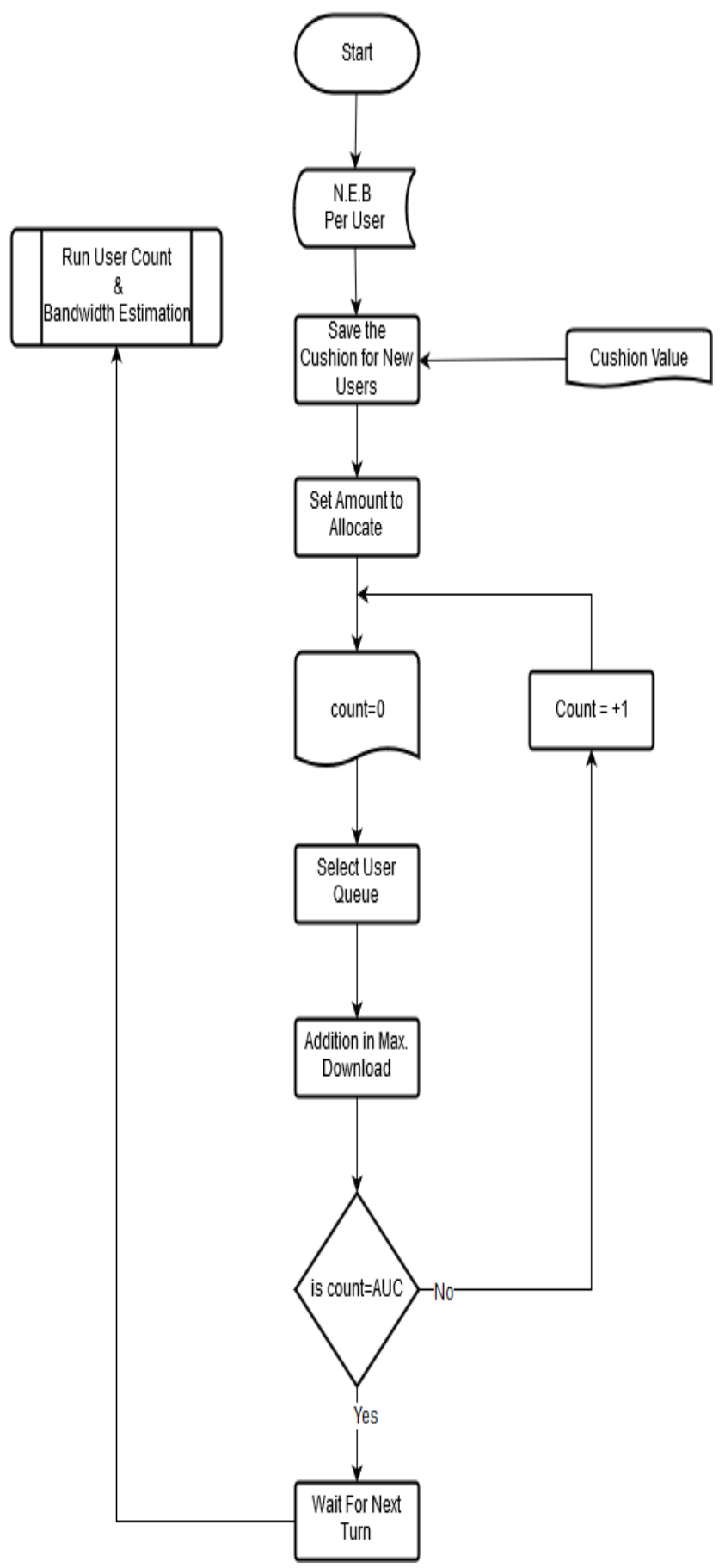

Fig. 3. Flow Chart for Excessive Bandwidth Allocation.

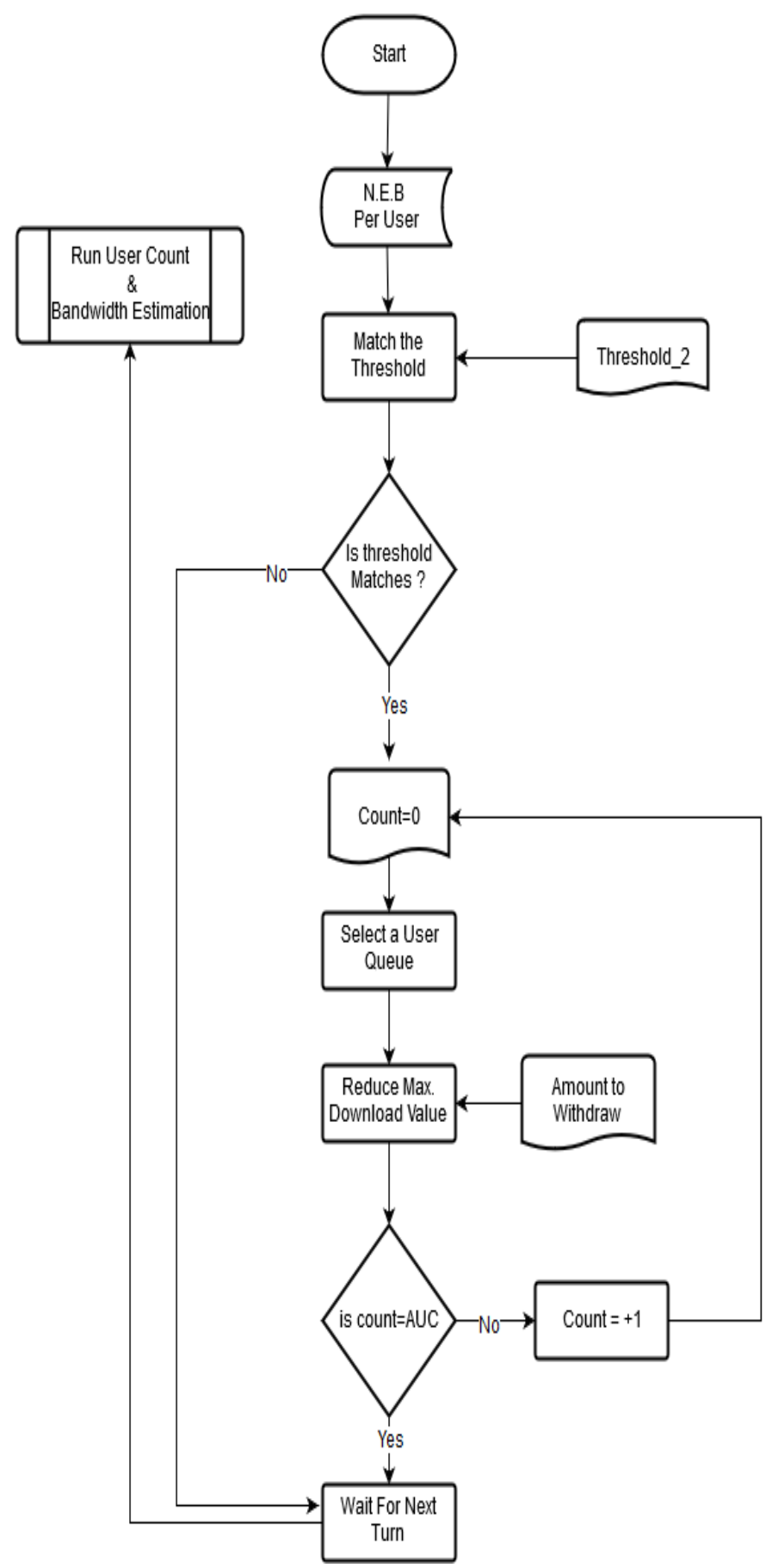

Fig. 4. Flow Chart for Withdrawal of Bandwidth. 


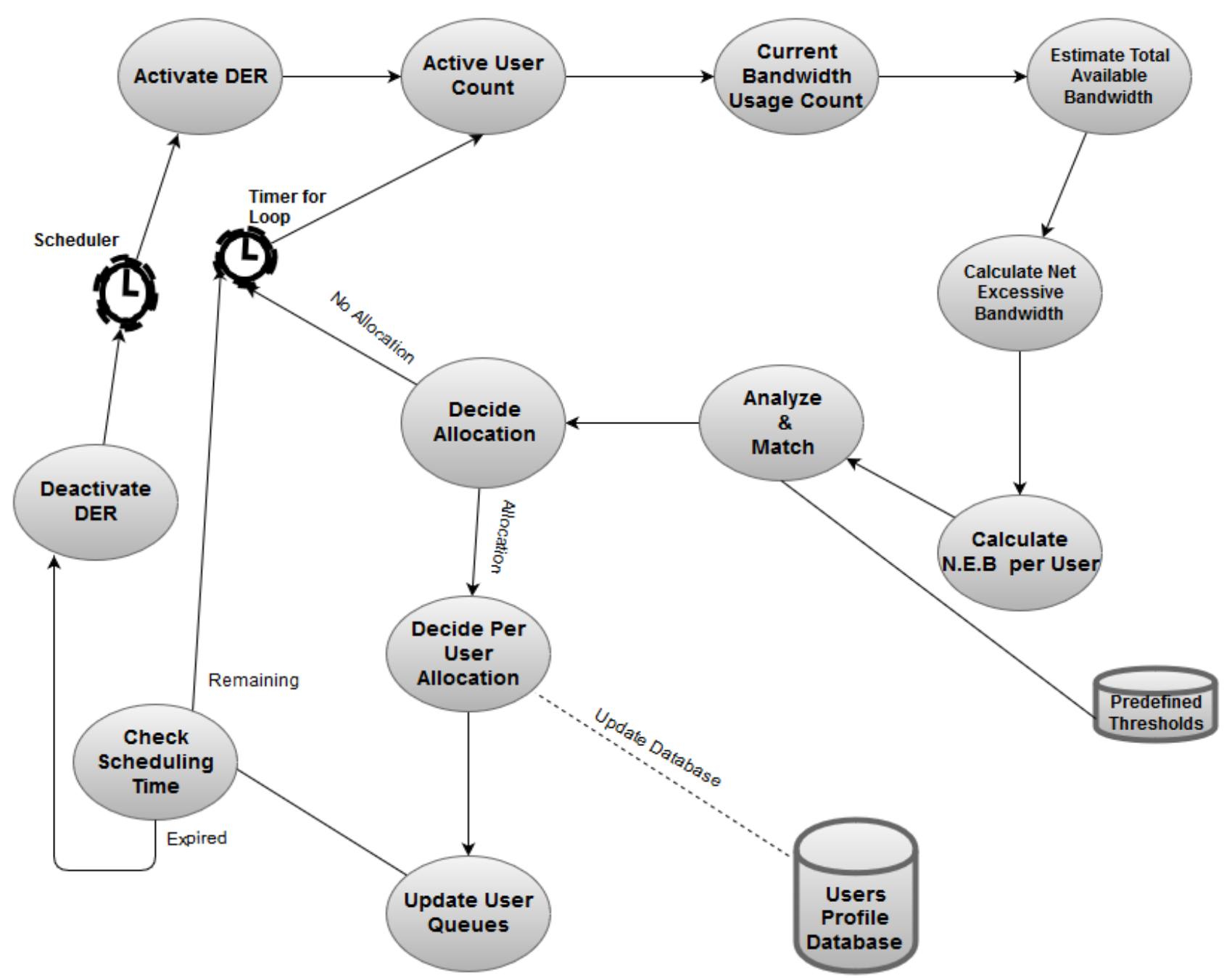

Fig. 5. DER Allocation Frame Work.

\section{CONCLUSION}

In this paper, different we have proposed a new and effective DBA scheme, that focuses on the efficient allocation of the excessive network bandwidth among the users, especially during the off-peak hours when network traffic in marginally low. Moreover, the objective is to allocate the excessive, but not to withdraw and increase the user satisfaction by providing some extra other than that they have paid for.

We further aim to practically implement DER on any local area network so that bandwidth monitoring can be observed for efficient bandwidth allocation to increase the user satisfaction. Moreover, by conducting different experiments results are expected to show that total available backbone bandwidth will be increased in great numbers. As far as limitations are concerned, the proposed scheme is only for large local area network.

\section{REFERENCES}

[1] Bisio, M. Marcliese, "Performance studies of bandwidth allocation technologies for QOS-constrained satellite networks", in IEEE international communication conference, Glasgow, 2007, pp.7-12
[2] J. Aracil, D. Morato, 'A-Priori Flow Bandwidth Estimates for Dynamic Bandwidth Allocation in ISP Access Links', in ITC Specialists Seminar on Access Networks and Systems, Gerona, Spain, 2001, p. 161-167.

[3] K. Kyeong Soo, 'On the excess bandwidth allocation in ISP traffic control for shared access networks', Communications Letters IEEE, vol.18, no.4, pp. 692-695, 2014.

[4] O. Haran, A. Sheffer, The Importance of Dynamic Bandwidth Allocation in GPON Networks', PMC-Sierra Incorporation, 2008.

[5] Satyajit Sarmah, Shikhar Kumar Sarma, "Dynamic Bandwidth Management in 802.11Wireless LAN", International Journal of Computer Sciences and Engineering, Vol.-6, Issue-6, June 2018.

[6] Xiaomei Yu, Doan B. Hoang, David D. Feng,"Weight-Based Fair Intelligent Bandwidth Allocation for Red Adaptive Video Traffic", 2006.

[7] Bo Fan, Supeng Leng, Kun Yang, "A dynamic bandwidth allocation algorithm in mobile networks with big data of users and networks", IEEE Network, Volume: 30, Issue: 1, January-February 2016.

[8] Mohammed Awad, Abdelmunem Abuhasan, "A Smart Clustering Based Approach to Dynamic Bandwidth Allocation in Wireless Networks", International Journal of Computer Networks \& Communications (IJCNC) Vol.8, No.1, January 2016.

[9] Elias, F. Martignon, A. Capone, "An Efficient Dynamic Bandwidth Allocation Algorithm for Quality of Service Networks".

[10] G. Xilouris, A. Kourtic ,G. Stefanou, " Dynamic bandwidth allocation for LAN2LAN interconnection using DVB-S satellite transmission", 2019. 